\title{
Ultrasonography and transillumination for uveal melanoma localisation in proton beam treatment planning
}

\author{
Jonathan E. Lu ${ }^{1} \cdot$ R. Joel Welch ${ }^{1} \cdot$ Kavita K. Mishra ${ }^{2} \cdot$ Inder K. Daftari ${ }^{2} \cdot$ Susanna S. Park ${ }^{1}$
}

Received: 21 October 2018 / Revised: 7 May 2019 / Accepted: 31 May 2019 / Published online: 5 July 2019

(c) The Author(s), under exclusive licence to The Royal College of Ophthalmologists 2019

\begin{abstract}
Background/Objective The success of proton beam treatment (PBT) in uveal melanoma depends in part on the accuracy of tumour localisation. This study determined if using ultrasonography (US) to measure the distance between tumour margin and tantalum ring (DTR) in PBT planning improves local treatment success when compared with using intraoperative transillumination (TI) alone.

Methods Retrospective analysis of patients with uveal melanoma treated at one centre between January 2006 and June 2017 with $\geq 12$-month follow-up (or until treatment failure). Local tumour control was compared among study groups based on methods for measuring DTR: Group 1 (TI alone), Group 2A (postoperative US alone) and Group 2B (combination).

Results Fifty-four eyes (54 patients) with uveal melanomas were included: Group 1 (22 eyes, 41\%), Group 2A (11 eyes, $20 \%$ ) and Group 2B (21 eyes, 39\%). Mean age at diagnosis was 64 years [median 66 years, range 23-86 years]. Fifty tumours (93\%) involved the choroid, while four involved the ciliary body (7\%). In Group 2B, PBT treatment was based on the DTR obtained using US; DTR differed between TI and US by $\geq 1 \mathrm{~mm}$ for 25 rings in 16 eyes and $\geq 2 \mathrm{~mm}$ for 12 rings in 7 eyes. Five-year Kaplan-Meier estimate revealed a difference in local treatment success between Groups 1 and $2,(0.82$ vs. $1.0, p=0.02)$ with no difference in overall survival estimate, $(0.85$ vs. $0.83, p=0.8)$.

Conclusions US can be used to measure DTR in PBT planning for uveal melanoma. This may improve accuracy of tumour localisation and improve local treatment success.
\end{abstract}

\section{Introduction}

Proton beam treatment (PBT) and plaque brachytherapy are the two local treatment options for uveal melanoma [1-4]. Local tumour control rates of $95 \%$ or greater have been reported in some clinical centres using the PBT, or brachytherapy combined with transpupillary thermotherapy [1-8]. Despite universally high reported rates of local tumour control following radiotherapy for uveal melanoma, the control rates do vary among centres. Opportunity exists to further refine radiation techniques to minimise the risks associated with over or under treatment.

Susanna S. Park

sscpark@ucdavis.edu

1 Department of Ophthalmology \& Vision Science, University of California Davis Health Eye Centre, Sacramento, CA, USA

2 Department of Radiation Oncology, University of California San Francisco, San Francisco, CA, USA
Optimising the accuracy of tumour localisation in PBT planning is one approach since approximately half of the tumour local recurrences after PBT occur at the tumour margin [1].

A standard step for PBT planning involves transillumination (TI) to measure the distance between tumour margin and sutured tantalum rings (DTR) [1,2]. Since DTR is used for PBT planning to determine how to aim the proton beam, the success of PBT would depend in part on the accuracy of DTR measurement. Along with DTR, the fundus photograph, ultrasound measurements, intraoperative surgical assessments, clinical appearance and tumour pigmentation are taken into consideration with PBT planning. While some have reported high accuracy in enucleated specimens examined for ring placement on the tumour border, this may not be consistently found across centres, and may vary depending on tumour sizes/locations and pigmentation [1]. In addition, some centres have incorporated additional imaging such as MRI and CT scanning to PBT planning to improve accuracy [9]. However, this is not standard of care practiced in all centres. 
Ultrasonography (US) is used routinely for uveal melanoma diagnosis and tumour size measurement. Historically, US has not been used for measuring DTR in PBT planning [1]. However, Daftari et al. [10] showed that US can aid in localising anterior margins of ciliary body tumours by detecting occult extension of the tumour into the ciliary body or iris which may not be evident using TI. Intraoperative US has been shown to improve accuracy of uveal melanoma localisation in plaque brachytherapy [11]. In this retrospective study, we assessed the feasibility of measuring DTR with US and the effect of incorporating US-derived DTR in PBT planning on PBT treatment outcomes including local treatment success and overall patient survival.

\section{Materials and methods}

This retrospective study reviewed the medical records of all patients diagnosed with uveal melanoma at the University of California Davis Eye Center and treated with PBT between January 2006 and June 2017. All patients with follow-up greater than 12 months at the study centre or until any local treatment failure were included. All patients had placement of tantalum rings and measurement of DTR using TI by one surgeon (SSP). Certified ocular ultrasonographers performed all US measurements for DTR. The study was performed in accordance with the United States Health Insurance Portability and Accountability Act of 1996 and study protocol approved by the University of California Office of Human Research. The research adhered to the tenets of the Declaration of Helsinki.

Data collected from medical records included patient demographic information, tumour characteristics (baseline dimensions, pigmentation, location), date of PBT, DTR measured by TI and/or US, duration of follow-up, local treatment failure and death. Tumour pigmentation was determined by SSP based on the fundus photographs taken prior to PBT. Local treatment failure was defined as tumour growth requiring repeat of radiotherapy and/or histopathologic evidence of live, actively dividing tumour cells after enucleation. The same radiation oncology team (KM, ID) conducted all proton beam irradiation.

Intraoperative TI alone was used to measure DTR in Group 1, while postoperative US alone was used in Group $2 \mathrm{~A}$, and a combination of TI and US was obtained in Group 2B. Group 2 represents the combination of Groups 2A and 2B. This US subset of the study group underwent US 1 week postoperatively to measure DTR. This was done initially at the discretion of the treating surgeon (SSP) to confirm the DTR measured intraoperatively with TI, but later done routinely. When TI failed to delineate tumour margins clearly due to lack of pigmentation, overlying subretinal fluid or posterior tumour location, localisation with indirect ophthalmoscopy was used for ring placement, but intraoperative DTR was not measured in these cases using TI (Group 2A). US was used as the sole method for determining DTR for PBT planning in these eyes. The DTR obtained by TI or US was used for PBT planning with preference for the DTR measurement obtained by US when both were obtained.

The ultrasound DTR measurements were obtained using the B-scan mode of the Aviso S ultrasound instrument (Quantel Medical, France) and a 10 or $20 \mathrm{mHz}$ probe $(20 \mathrm{mHz}$ probe used when available during the last year of study), The sutured tantalum rings were identified as a hyper-echogenic focus on the outer surface of the eye wall with hyper-echogenic shadowing, best visualised when gain is decreased by about $30 \%$ of optimal tumour visualisation (Fig. 1). The DTR was determined by using a calliper to measure the distance between the ring margin to the tumour margin closest to the ring. The measurements were obtained during the 1 week postoperative visit to allow time for the eye to recover from surgery. The DTR information was sent to the radiation oncologist to use for PBT planning.

All data were tabulated on Microsoft Excel 2016 and comparative analyses were performed regarding demographics, tumour location, tumours features and outcomes. Measures of central tendencies (mean, median and range) were obtained using built in functions. Independent twotailed student's $t$-test and analysis of variance test were used to analyse continuous data, while Fisher's exact test or $\chi^{2}$ test was used for categorical data. Five-year Kaplan-Meier analysis was performed using Microsoft Excel 2016 by grouping censored and outcome data into half-month intervals. Log-rank test was used to assess statistical significance among Kaplan-Meier data. A $p$-value less than 0.05 was considered statistically significant for all tests.

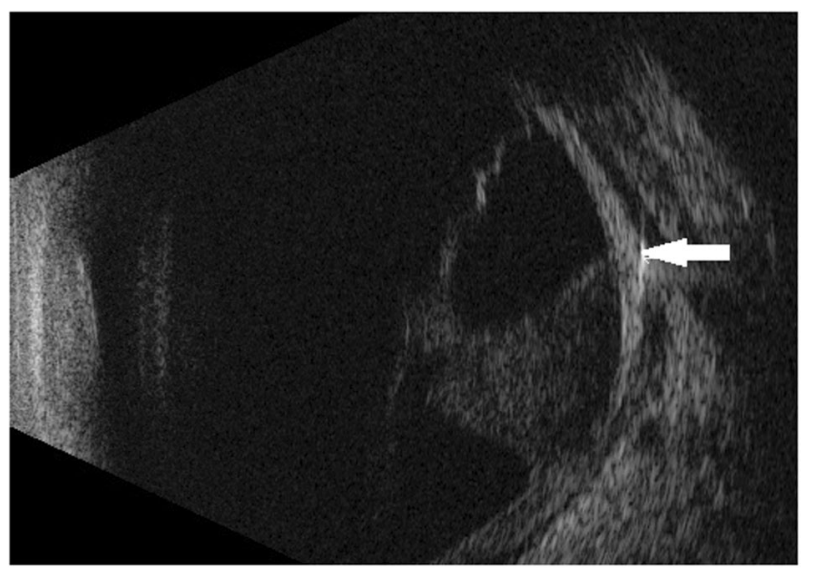

Fig. 1 Ultrasound B-scan image showing the position of the sutured tantalum ring relative to the tumour margin. The white arrow points to a focal hyperechogenic line with shadowing on the outer surface of the eye wall consistent with the sutured tantalum ring, which is at the tumour margin 


\section{Results}

Fifty-four eyes (54 patients) with uveal melanoma had PBT between January 2006 and June 2017 and follow-up of greater than 12 months (or treatment failure sooner) at the study centre. The study group included 27 males (50\%) and 27 females (50\%). Demographic and tumour characteristics of the study group are summarised in Table 1 . Mean age at diagnosis was 64 years [median 66 years, range 23-86 years]. Fifty uveal tumours localised to the choroid (93\%) and four tumours $(7 \%)$ involved the ciliary body. Mean largest basal diameter was $10.7 \mathrm{~mm}$ [median $10.4 \mathrm{~mm}$, range $4.1-21.1 \mathrm{~mm}$ ] while mean thickness was $4.0 \mathrm{~mm}$ [median $2.8 \mathrm{~mm}$, range $1.7-14.3 \mathrm{~mm}$ ].

Intraoperative TI alone was used to measure DTR in 22 eyes (Group 1,41\%), while postoperative US alone was used 11 eyes (Group 2A, 20\%), and a combination of TI and US was obtained in 21 eyes (Group 2B, 39\%). Four tantalum rings were placed typically for each eye totalling 214 rings (two eyes had only three rings). There was no difference in baseline demographic or tumour characteristics between these groups. The mean tumour to ring distance by TI technique across groups was $-0.018 \mathrm{~mm}$ (median $0 \mathrm{~mm}$, range -5 to $5 \mathrm{~mm}$, interquartile range -1 to $1 \mathrm{~mm})$. The mean DTR by ultrasound technique across groups was $0.18 \mathrm{~mm}$ (median 0 , range -7.87 to $4.36 \mathrm{~mm}$, interquartile range -0.23 to $1.23 \mathrm{~mm}$ ).

Among 32 eyes in Group 2 (Group 2A and 2B combined), all sutured tantalum rings could be visualised readily postoperatively using US (Fig. 1), such that DTR could be readily obtained in all eyes. Among these eyes, PBT planning was based on DTR obtained using US. Among 21 eyes that had DTR measured using both methods (Group 2B), the mean absolute difference in DTR measurements between TI and US was $0.9 \mathrm{~mm}$ [median $0.6 \mathrm{~mm}$, range 0-4.4 mm]. Measurements for DTR differed between TI and US by $\geq 1 \mathrm{~mm}$ for 25 rings in 16 eyes and $\geq 2 \mathrm{~mm}$ for 12 rings in 7 eyes. Table 2 compares Group 2B tumour and ring characteristics in eyes where there was at least one ring with DTR difference of $\geq 2 \mathrm{~mm}$ between the TI and US measurements. Only largest tumour basal diameter was different between the smaller and larger DTR groups (13.9 $\mathrm{mm}$ vs. $10.3 \mathrm{~mm}, p=0.002$ ). In contrast, tumour thickness $(p=0.2)$, tumour location $(p=0.9)$, tumour pigmentation $(p=0.4)$ and distance of ring to limbus $(p=0.5)$ were not significant factors affecting differences in DTR between methods.

Follow-up and outcome data are summarised in Table 3. Overall local tumour control (all groups) was 93\% during mean follow-up of 62 months [median 60 months, range

Table 1 Demographics stratified by the measurement method for distance between tumour margin and tantalum ring (DTR)

\begin{tabular}{|c|c|c|c|c|c|}
\hline \multirow[b]{3}{*}{ Feature } & \multicolumn{3}{|c|}{ DTR measurement method } & \multirow[b]{3}{*}{$p$-value ${ }^{\mathrm{a}}$} & \multirow[b]{3}{*}{$\begin{array}{l}\text { Total } \\
{[n=54 \text { patients }]}\end{array}$} \\
\hline & Group 1 & \multicolumn{2}{|l|}{ Group 2} & & \\
\hline & $\begin{array}{l}\text { Transillumination only } \\
{[n=22 \text { patients }]}\end{array}$ & $\begin{array}{l}\text { Group } 2 \mathrm{~A} \\
\text { Ultrasound only } \\
{[n=11 \text { patients }]}\end{array}$ & $\begin{array}{l}\text { Group 2B } \\
\text { Transillumination and } \\
\text { ultrasound } \\
{[n=21 \text { patients }]}\end{array}$ & & \\
\hline Age mean, years [median, range] & $68[67,44-86]$ & $60[65,27-76]$ & $62[63,23-81]$ & 0.2 & $64[66,23-86]$ \\
\hline Sex, no. $(\%)$ & & & & $0.5^{\mathrm{b}}$ & \\
\hline Male & $9(41)$ & $6(55)$ & $12(57)$ & & $27(50)$ \\
\hline Female & $13(59)$ & $5(45)$ & $9(43)$ & & $27(50)$ \\
\hline Laterality, no. (\%) & & & & $0.3^{\mathrm{b}}$ & \\
\hline Right & $16(73)$ & $5(45)$ & $14(67)$ & & $35(65)$ \\
\hline Left & $6(27)$ & $6(55)$ & $7(33)$ & & $19(35)$ \\
\hline \multicolumn{6}{|l|}{ Tumour size } \\
\hline Mean base, mm [median, range] & $10.8[10.5,4.8-16.2]$ & $8.9[9.1,4.1-13.7]$ & $11.5[11.1,5.5-21.1]$ & 0.1 & $10.7[10.4,4.1-21.1]$ \\
\hline $\begin{array}{l}\text { Mean thickness, mm } \\
\text { [median, range] }\end{array}$ & $3.9[2.9,1.7-13.1]$ & $2.9[2.3,1.9-8.7]$ & $4.8[3.2,1.7-14.3]$ & 0.2 & $4.0[2.8,1.7-14.3]$ \\
\hline Tumour epicentre location & & & & $0.054^{\mathrm{c}}$ & \\
\hline Macula & $3(14)$ & $6(55)$ & $2(10)$ & & $11(21)$ \\
\hline Macula to equator & $7(31)$ & $4(36)$ & $11(52)$ & & $22(41)$ \\
\hline Equator to ora serrata & $9(41)$ & $1(9)$ & $7(33)$ & & $17(31)$ \\
\hline Ciliary body & $3(14)$ & 0 & $1(5)$ & & $4(7)$ \\
\hline Tumour pigment, $\%$ [median, range] & $67[70,5-100]$ & $67[80,5-100]$ & $65[70,15-100]$ & 0.9 & $66[70,5-100]$ \\
\hline
\end{tabular}

DTR distance between tumour margin and tantalum ring

andependent $t$-test

${ }^{\mathrm{b}}$ Chi-squared test

${ }^{\mathrm{c}}$ Fisher exact test 
Table 2 Tumour and ring characteristics stratified by difference in distance between tumour margin and tantalum ring (DTR) for tumours where DTR was measured by both transillumination and ultrasound, Group 2B

\begin{tabular}{|c|c|c|c|c|}
\hline \multicolumn{5}{|l|}{ Group 2B } \\
\hline \multirow[t]{2}{*}{ Feature } & $\begin{array}{l}\text { All tantalum rings with }<2 \mathrm{~mm} \\
\text { difference in DTR }\end{array}$ & $\begin{array}{l}\text { At least } 1 \text { tantalum ring per tumour with } \\
\geq 2 \mathrm{~mm} \text { difference in DTR }\end{array}$ & $p$-value ${ }^{\mathrm{a}}$ & Total \\
\hline & [ $n=14$ tumours, 25 rings $]$ & {$[n=7$ tumours, 12 rings $]$} & & {$[n=21$ tumours $]$} \\
\hline \multicolumn{5}{|l|}{ Tumour size } \\
\hline $\begin{array}{l}\text { Mean base, } \mathrm{mm} \\
\text { [median, range] }\end{array}$ & $10.3[10.3,5.5-14.6]$ & $13.9[13.9,7.8-21.1]$ & 0.047 & $10.7[10.4,4.1-21.1]$ \\
\hline $\begin{array}{l}\text { Mean thickness, mm } \\
\text { [median, range] }\end{array}$ & $4.0[3.0,1.7-11.9]$ & $6.4[5.6,2.0-14.3]$ & 0.2 & $4.0[2.8,1.7-14.3]$ \\
\hline \multicolumn{5}{|l|}{$\begin{array}{l}\text { Tumour epicentre } \\
\text { location }(\%)\end{array}$} \\
\hline Macula & $2(14)$ & 0 & & $11(20)$ \\
\hline Macula to equator & $7(50)$ & $4(57)$ & $0.9^{\mathrm{b}}$ & $22(41)$ \\
\hline Equator to ora serrata & $4(29)$ & $3(43)$ & & $17(31)$ \\
\hline Ciliary body & $1(7)$ & 0 & & $4(7)$ \\
\hline $\begin{array}{l}\text { Tumour pigment, } \% \\
\text { [median, range] }\end{array}$ & $62[65,15-90]$ & $71[70,40-100]$ & 0.4 & $66[70,5-100]$ \\
\hline $\begin{array}{l}\text { Mean distance of ring to } \\
\text { limbus, mm [median, range] }\end{array}$ & $14.1[14.8,0.5-21]$ & $14.7[14.8,4.0-20]$ & 0.5 & $14.3[14.8,0.5-21]$ \\
\hline
\end{tabular}

TI transillumination, US ultrasound, DTR distance between tumour margin and tantalum ring

${ }^{\mathrm{a}}$ Independent $t$-test

${ }^{\mathrm{b}}$ Fisher exact test

Table 3 Follow-up, local treatment failure and 5-year Kaplan-Meier estimates based on the method of distance between tumour margin and tantalum ring (DTR) measurement

\begin{tabular}{|c|c|c|c|c|}
\hline Feature & $\begin{array}{l}\text { Group } 1 \\
{[n=22 \text { patients }]}\end{array}$ & $\begin{array}{l}\text { Group } 2 \\
{[n=32 \text { patients }]}\end{array}$ & $p$-value ${ }^{\mathrm{a}}$ & $\begin{array}{l}\text { Total } \\
{[n=54 \text { patients }]}\end{array}$ \\
\hline $\begin{array}{l}\text { Mean follow-up, months } \\
\text { [median, range] }\end{array}$ & $80[86,6-134]$ & $50[49,12-123]$ & 0.002 & $62[60,6-134]$ \\
\hline Local treatment failure $(\%)$ & $4(18)$ & $0(0)$ & $\mathbf{0 . 0 2}{ }^{\mathrm{b}}$ & $4(7)$ \\
\hline $\begin{array}{l}\text { Interval to local treatment failure, } \\
\text { months [median, range] }\end{array}$ & $12[10,5-23]$ & na & na & $12[10,5-23]$ \\
\hline \multicolumn{5}{|l|}{ 5-year KM estimate for: } \\
\hline Local treatment success & 0.82 & 1 & $\mathbf{0 . 0 2 ^ { \mathrm { c } }}$ & 0.92 \\
\hline Overall survival & 0.85 & 0.83 & $0.8^{\mathrm{c}}$ & 0.84 \\
\hline
\end{tabular}

na not applicable, KM Kaplan-Meier, DTR distance between tumour margin and tantalum ring

andependent $t$-test

${ }^{\mathrm{b}}$ Fisher exact test

${ }^{\mathrm{c}}$ Log-rank test
6-134 months]. Four local treatment failures were noted during follow-up, all from Group 1, with mean interval to failure of 12 months [median 10 months, range 5-23 months]. Patient 1 had a posterior tumour with $100 \%$ pigmentation. The eye was enucleated 6 months after proton beam for marked increase in tumour size with tumour breaking through Bruch's membrane. Histology of the enucleated eye showed actively dividing melanoma cells. Patient 2 had a peripheral tumour with $40 \%$ pigmentation. Recurrent tumour growth was noted at the margin of the original melanoma at 23 months follow-up. The eye was re-treated with PBT with local control at last follow-up. This patient passed away 5 years after initial PBT from metastatic complications. Patient 3 had a posterior choroidal melanoma with variable pigmentation and extensive overlying exudative retinal detachment. At 9 months after PBT, increasing tumour dimension and subretinal fluid was noted. The eye was enucleated and histology showed live dividing melanoma cells. Patient 4 had a posterior choroidal melanoma with $70 \%$ pigmentation. Tumour recurrence was diagnosed 1 year after PBT. Enucleation was performed and histology confirmed diagnosis. 
In contrast, no local failure was noted in Groups $2 \mathrm{~A}$ and 2B (collectively Group 2) after mean follow-up of 50 months [median 49 months, range 12-123 months]. Five-year Kaplan-Meier estimate revealed a difference in local treatment success between Groups 1 and 2 (0.82 vs. $1.0, p=0.02$ ) with no difference in overall survival estimate (0.85 vs. $0.83, p=0.8$ ) (Fig. 2 ).

\section{Discussion}

In this retrospective study, we found that sutured tantalum rings for PBT planning could be readily visualised postoperatively by US in all eyes where this was attempted. When comparing eyes treated with proton beam using the US-derived DTR measurements for treatment planning (Group 2) with those eyes treated with proton beam using the traditional TI method to determine DTR for treatment

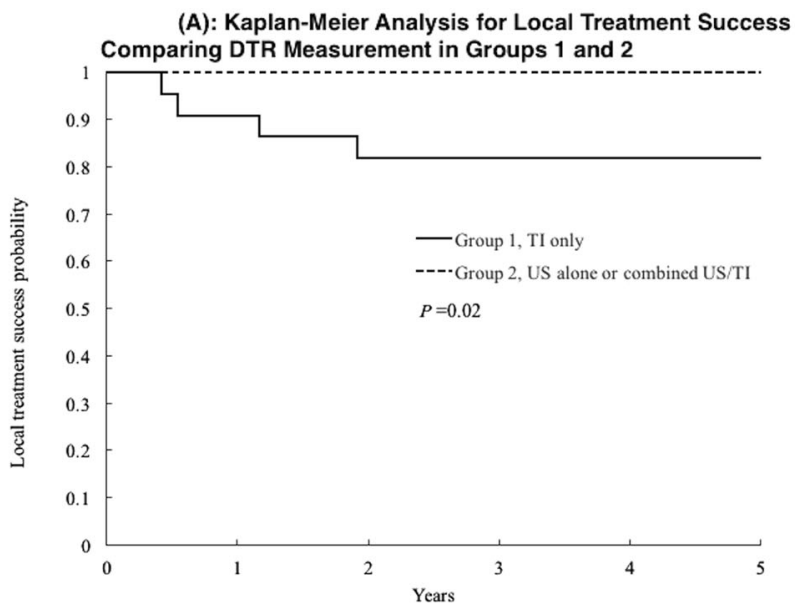

(B): Kaplan-Meier Analysis for Overall Survival Comparing

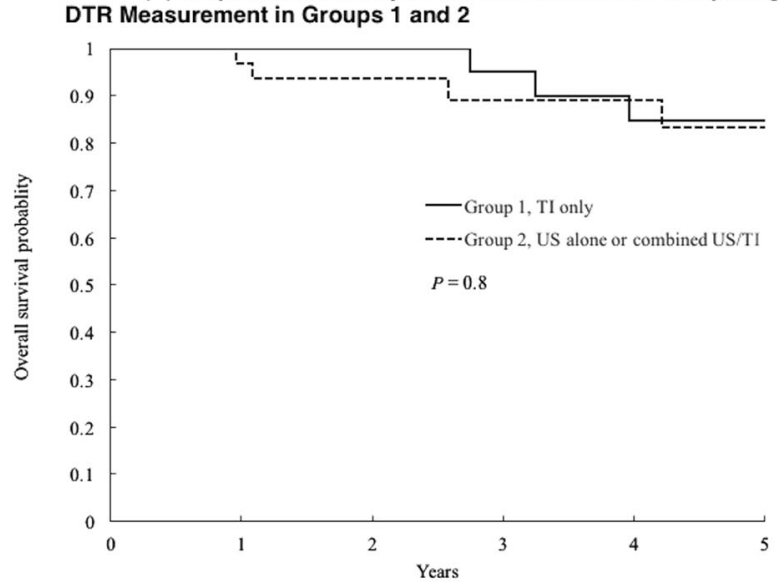

Fig. 2 Kaplan Meier probability of local treatment success and overall survival between study groups over time. a Local tumour treatment success rate over time. b Overall survival over time. DTR $=$ distance between tumour margin and tantalum ring; $\mathrm{TI}=$ transillumination; $\mathrm{US}=$ ultrasound planning (Group 1), local tumour control following PBT was statistically better in the group treated with proton beam using US-derived DTR. In a subset of 83 rings, where tumour localisation was conducted using both TI and US, Group 2B, differences in DTR of $2 \mathrm{~mm}$ or greater were noted for 12 rings in 7 eyes. Although PBT planning generally accounts for an additional 2-2.5-mm border beyond the tumour margin, the observed difference in DTR could be clinically significant as the dose of radiation delivered by PBT is very localised with minimal lateral spread of radiation beyond $1-2 \mathrm{~mm}$ of the target [1-3].

Tumour features were analysed for possible effect on the difference in DTR measurements obtained using US vs. TI. Surprisingly, tumour pigmentation was not found to be a significant factor although low tumour pigmentation can make tumour localization challenging using TI. Selection bias may have had a role as the treating surgeon commonly used US only when measuring DTR in minimally pigmented tumours. Among eyes that had both imaging modalities to determine DTR (Group 2B), increasing tumour basal diameter was found to be a significant risk factor for increased discrepancy in DTR between methods, with a larger basal diameter yielding a higher absolute DTR difference. This could be from pigment variations at the tumour margin that TI may not capture. Shadowing effects from TI in eyes with larger tumours may also be contributory, although one may expect this to be more attributable to tumours with increased thickness, rather than increased basal diameter.

One subject in Group 1 had DTR obtained with both methods but PBT was planned using DTR obtained with TI only. This subject was the first patient on whom US was used to determine feasibility of obtaining DTR using this method. Although a discrepancy in DTR between US and TI was noted in this case, the US information was obtained too late to be incorporated into the PBT planning and treatment. This patient was one of the local treatment failures (Patient 3) and the outcome of this patient was part of the rationale for conducting this study. Currently, the DTR obtained by US is used routinely for PBT planning at our centre and $100 \%$ local control has been maintained.

In this retrospective study, the mean follow-up period for Group 2 was shorter than Group 1. Prior reports have shown that the majority of local treatment failures following PBT occur in the first 1-2 years [2, 3]. This timeline is consistent with our study observations as all four local tumour recurrences occurred within 23 months of treatment. While the mean follow-up interval was shorter for Group 2, it was longer than the 1- to 2-year interval during which local recurrence is usually seen.

There are several limitations to our study. First, the sample size was limited and may not be powered to detect 
all factors that affect the accuracy of DTR measurement and success of PBT. Nevertheless, we were able to identify increasing tumour basal diameter as a tumour feature associated with increased discrepancy in DTR obtained using the two methods. While local tumour control rate for Group 1 was lower than other reports, the sample size of this single centre study was limited and the overall tumour control rate of this study was comparable to rates reported by larger centres $[2,3]$. In some uveal melanomas with minimally elevated borders (diffuse configuration), one might theorise that TI may be preferred over ultrasound for obtaining DTR. In fact, since sonographers use the sharp change in echogenic signal within the eye wall as sonographic landmark of tumour margin for baseline diameter measurements for all choroidal melanomas, the accuracy of DTR determination by sonography should not be affected by low tumour height. Nevertheless, US technique and the experience of the sonographer may have an impact on the visualisation plane and hence the final measurement outcomes. Hence, we suggest the use of both techniques of TI and US whenever possible. Finally, the decision to perform US to obtain DTR was initially at the discretion of the treating ocular oncologist (SSP); thus, selection bias may apply.

Initially, the decision to use US for DTR measurement was based on factors that might adversely affected visualisation of tumour margins by TI, e.g. posterior tumour location, minimal/variable tumour pigmentation. Currently at this study centre, US is performed routinely to obtain DTR after tantalum ring placement. As a result, the TI group (Group 1) tended to include eyes that were treated earlier than Group 2 eyes and it is possible that the latter cohort benefitted from additional experience at the study centre. Although a "cohort" effect cannot be ruled out, it is an unlikely explanation for the difference in treatment outcome, since a difference in DTR continued to be noted frequently between methods when both were used. While not directly comparable, the observed rate of difference in tumour localization between the techniques noted in our study is similar to the difference noted in brachytherapy plaque placement [11]. Given the rise in access to proton treatment and ophthalmic centres involved in tantalum ring placement, ultrasound may provide additional data to ensure accuracy and reproducibility of DTR measurements, potentially affecting local control and dose-dependent radiation-related side effects $[12,13]$.

Other methods to improve tumour localization for PBT planning have been explored in some centres, including the use of MRI and CT scan of the orbit [9]. Although our study did not compare the relative accuracy of US to MRI or CT scan imaging, the clinical outcome after incorporating US to determine DTR has been excellent. Furthermore, US offers many advantages over MRI and/or CT scanning in terms of time, cost, radiation exposure and accessibility.
In summary, this is the first study to demonstrate the feasibility of using US measurement to determine DTR in PBT planning for uveal melanoma. Local tumour control may be improved when US-derived DTR is incorporated into PBT.

\section{Summary}

\section{What was known before}

- Irradiation beam therapy for ocular melanomas requires accurate localization of tumour for effective treatment. Ultrasound is frequently used in the diagnosis and monitoring of intraocular tumours, but has not been used to guide radiation treatment planning.

\section{What this study adds}

- This study is the first demonstration that ultrasound, a relatively affordable, widely available and safe modality of imaging, can improve ocular melanoma treatment outcomes by improving localization for radiation therapy.

Acknowledgements The authors thank Ellen Redenbo and Karishma Chandra for their help with ultrasonography. The study was presented in part as a poster at the annual meeting of the American Academy of Ophthalmology, 15 November 2015, Las Vegas, NV and as a poster at the annual meeting Association for Research in Vision and Ophthalmology, Denver, CO, 7 May 2015.

Funding This work was supported in part by the University of California Davis Medical Student Research Award (JEL).

\section{Compliance with ethical standards}

Conflict of interest The authors declare that they have no conflict of interest.

Publisher's note: Springer Nature remains neutral with regard to jurisdictional claims in published maps and institutional affiliations.

\section{References}

1. Gragoudas ES. Proton beam irradiation of uveal melanomas: the first 30 years. The Weisenfeld Lecture. Investig Ophthalmol Vis Sci. 2006;47:4666-73.

2. Gragoudas ES, Li W, Goitein M, Lane AM, Munzenrider JE, Egan KM. Evidence-based estimates of outcomes in patients irradiated for intraocular melanoma. Arch Ophthalmol. 2002; 120:1665-71.

3. Castro JR, Char DH, Petti PL, Daftari IK, Quivey JM, Singh RP, et al. 15 years experience with helium ion radiotherapy for uveal melanoma. Int J Rad Oncol Biol Phys. 1997;39:989-96. 
4. Seddon JM, Gragoudas ES, Egan KM, Glynn RJ, Howard S, Fante RG, et al. Relative survival rates after alternative therapies for uveal melanoma. Ophthalmology 1990;97:769-77.

5. Shields CL, Furuta M, Thangappan A, Nagori S, Mashayekhi A, Lally DR, et al. Metastasis of uveal melanoma millimeter-bymillimeter in 8033 consecutive eyes. Arch Ophthalmol. 2009;127:989-98.

6. Shields CL, Cater J, Shields JA, Chao A, Krema H, Materin M, et al. Combined plaque radiotherapy and transpupillary thermotherapy for choroidal melanoma: tumor control and treatment complications in 270 consecutive patients. Arch Ophthalmol. 2002;120:933-40.

7. Collaborative Ocular Melanoma Study Group. The COMS randomized trial of iodine 125 brachytherapy for choroidal melanoma: V. Twelve-year mortality rates and prognostic factors: COMS Report No. 28. Arch Ophthalmol. 2006;124:1684-93.

8. Mishra KK, Quivey JM, Daftari IK, Weinberg V, Cole TB, Patel $\mathrm{K}$, et al. Long-term results of the UCSF-LBNL randomized trial: charged particle with helium ion versus iodine-125 plaque therapy for choroidal and ciliary body melanoma. Int J Radiat Oncol Biol Phys. 2015;92:376-83.
9. Marnitz S, Cordini D, Bendl R, Lemke AJ, Heufelder J, Simiantonakis I, et al. Proton therapy of uveal melanomas: intercomparison of MRI-based and conventional treatment planning. Strahl Onkol. 2006;182:395-9.

10. Daftari I, Barash D, Lin S, O'Brien J. Use of high-frequency ultrasound imaging to improve delineation of anterior uveal melanoma for proton irradiation. Phys Med Biol. 2001;46:579-90.

11. Chang MY, Kamrava M, Demanes DJ, Leu M, Agazaryan N, Lamb J, et al. Intraoperative ultrasonography-guided positioning of iodine 125 plaque brachytherapy in the treatment of choroidal melanoma. Ophthalmology 2012;119:1073-77.

12. Mishra KK, Daftari IK, Weinberg V, Cole T, Quivey JM, Castro $\mathrm{JR}$, et al. Risk factors for neovascular glaucoma after proton beam therapy of uveal melanoma: a detailed analysis of tumor and dose-volume parameters. Int $\mathbf{J}$ Radiat Oncol Biol Phys. 2013;87:330-6.

13. Polishchuk AL, Mishra KK, Weinberg V, Daftari IK, Nguyen JM, Cole $\mathrm{T}$, et al. Temporal evolution and dose-volume histogram predictors of visual acuity after proton beam radiation therapy of uveal melanoma. Int J Radiat Oncol Biol Phys. 2017;97:91-97. 